\title{
Pratiques
}

Linguistique, littérature, didactique

187-188| 2020

Enseignement du texte littéraire dans l'espace

francophone: pratiques, formation, recherche

\section{L'explication de texte et ses avatars : des exercices en tension dans les programmes}

Text Explanation and its Avatars: Tensions at Work in the Curriculum

\section{Laetitia Perret}

\section{(2) OpenEdition \\ Journals \\ Édition électronique \\ URL : https://journals.openedition.org/pratiques/8812 \\ DOI : $10.4000 /$ pratiques.8812 \\ ISSN : 2425-2042 \\ Éditeur \\ Centre de recherche sur les médiations (CREM) \\ Référence électronique \\ Laetitia Perret, «L'explication de texte et ses avatars : des exercices en tension dans les \\ programmes ", Pratiques [En ligne], 187-188|2020, mis en ligne le 12 décembre 2020, consulté le 21 \\ juillet 2021. URL : http://journals.openedition.org/pratiques/8812 ; DOI : https://doi.org/10.4000/ pratiques.8812}

Ce document a été généré automatiquement le 21 juillet 2021.

(c) Tous droits réservés 


\title{
L'explication de texte et ses avatars : des exercices en tension dans les programmes
}

\author{
Text Explanation and its Avatars: Tensions at Work in the Curriculum
}

Laetitia Perret

Nombre d'articles dans ce numéro soulignent que la lecture analytique est un exercice en tension entre lecture participative et primat donné aux analyses. Il s'agira dans cet article d'interroger l'origine de ces tensions, dans une perspective historico-didactique (Bishop, 2017), en se limitant aux textes officiels français ${ }^{1}$ pour le lycée ${ }^{2}$. En effet, les instructions officielles (IO) « quand bien même elles représentent un curriculum formel ou virtuel par rapport à la réalité des classes, dessinent les traits principaux de la discipline français» (Petitjean \& Privat, 1999, p.6), elles permettent donc de comprendre la place qu'occupe l'exercice dans la culture disciplinaire.

2 Nous partons de l'hypothèse qu'une des causes de ces tensions est la dimension métatextuelle de l'exercice, présente dès l'origine (1880) et qui perdure dans la lecture méthodique (1987) et la lecture analytique (2001). En effet, dans la perspective qu'adopte B. Daunay (2005, p. 51-52) à propos du commentaire, l'explication de texte et ses avatars sont, comme le commentaire et la dissertation.

un genre du discours métatextuel qui peut se réaliser en exercices divers, qui rendraient tous compte d'une activité métatextuelle. Et ce genre se définirait précisément par son ouverture, par son hétérogénéité, par son évolution historique et aussi par sa labilité dans les pratiques de classe.

3 Le terme, d'avatar, au sens propre, désigne les changements d'apparence d'un individu - ou d'un dieu - dont l'essence perdure. En didactique il est utilisé pour désigner la part de permanence et de renouvèlement des objets scolaires ${ }^{3}$. L'approche métatextuelle est-elle l'essence qui perdure dans l'explication de texte? Qu'est-ce qui change, se transforme, dans cet exercice, et en quoi ces permanences/changements génèrent-ils des tensions? 
4 Pour répondre à cette question nous avons organisé notre réponse en trois temps, en suivant une progression chronologique qui met au jour différentes tensions. La première est liée à l'approche scientifique des textes qui fonde l'exercice en 1880 , ce qui a pour conséquences une lecture métatextuelle, une place importante accordée à la méthodologie et la méfiance à l'égard des impressions personnelles. La deuxième réside dans les programmes eux-mêmes qui déplorent une lecture standardisée, sans pour autant remettre en question l'approche métatextuelle, dans un exercice qui s'est réifié. La troisième se met en place dans les années 1980, lorsque les deux théories de référence qui sous-tendent l'exercice (grammaire, histoire littéraire) sont reconfigurées, et qu'en apparait une troisième, celle du sujet lecteur, alors que l'exercice continue de se réclamer d'une approche méthodique.

\section{Légitimer l'enseignement de la littérature : aux origines de l'exercice}

5 L'essence de l'explication de texte, que conserveront tous ses avatars, est bien sa dimension métatextuelle qui suppose une pratique de commentaire à forte dimension méthodologique, à travers une observation des textes dans un esprit scientifique. Si l'exercice évolue au gré de sédimentations successives (Schneuwly, 2007), cette approche conserve des constantes dont un des points communs est l'exclusion de toute implication personnelle.

\section{Légitimer l'enseignement de la littérature par l'approche méthodique}

6 La création de l'explication de texte ne peut être dissociée de l'invention de la discipline "littérature » au lycée, pour reprendre le titre de l'ouvrage de Martine Jey (1998). Cette dernière rappelle aussi le rôle de G. Lanson dans cette mise en place, auquel nous ferons référence. Légitimer la littérature en 1880, c'est la distinguer de la rhétorique. Nombre de recherches ont éclairé ce moment et nous en résumons ici les principaux apports à notre discussion. Tout d'abord, le corpus s'élargit à de nouveaux auteurs, de nouveaux siècles (Chervel, 2006 ; Jey, 1998). Ensuite, l'histoire littéraire devient, comme le notent les programmes de 1909, «Le lien nécessaire, le fond commun des lectures particulières; [...]» (Jey, 1998, p. 69). Enfin, on passe d'une conception scripturale à une conception lectorale de l'enseignement des textes littéraires (Denizot, 2013b). On ne les lit plus pour les imiter, via des exercices comme le discours, mais pour les expliquer. Les IO de 1909 ont de ce point de vue une formule intéressante: "L'essentiel est bien la lecture, éducatrice de l'attention, de l'esprit de précision et de méthode, et, à certains égards, du caractère même » (Jey, 1998, p. 69). Si la lecture est bien désormais au cœur de la culture disciplinaire, c'est non seulement pour former le goût (comme à l'époque de l'enseignement rhétorique, nous y reviendrons) mais aussi parce qu'elle nécessite une méthode, permet d'être précis, ce qui est une nouveauté.

7 Cette formule montre aussi tout ce que cette nouvelle configuration disciplinaire doit à l'héritage positiviste. En effet, à la fin du xix ${ }^{\mathrm{e}}$ siècle «la référence à la science est le passage obligé de toute création ou de toute réflexion littéraire » (Delfau, 1978, p. 235), dans l'enseignement secondaire comme dans le supérieur (Bompaire-Evesque, 2002). C'est parce que l'histoire littéraire, à travers la démarche historique, revendique une 
dimension scientifique qu'elle se scolarise, comme l'explique Lanson (1965 [1925], p. 31) : «Si mes réflexions regardent principalement la littérature française depuis la Renaissance, c'est que je la connais mieux et j'y pense constamment : c'est aussi que dans tous les autres domaines, on ne conteste pas l'utilité des méthodes exactes ». Cette approche scientifique va se traduire par l'importance accordée à la méthodologie. La littérature, pour légitimer son statut scolaire et devenir une discipline, doit adopter une méthode expérimentale. Cela va avoir des conséquences durables sur les pratiques de lecture scolaire des textes littéraires (approche métatextuelle, importance de la méthodologie) et nuire tout aussi durablement à l'expression de la subjectivité du lecteur.

\section{Approche métatextuelle, méthodologie et refus de l'impressionnisme}

8 L'approche métatextuelle des textes qui se met alors en place est une conséquence de cette légitimation de l'approche méthodologico-scientifique. Après 1880 , les exercices au lycée n'ont officiellement plus vocation à reproduire la belle page, à rechercher l'ornement, comme c'était le cas à l'époque de l'enseignement rhétorique, mais à la commenter. G. Genette (1982, p. 11) définit la métatextualité comme « La relation, on dit plus couramment de "commentaire", qui unit un texte à un autre texte dont il parle [...]. C'est par excellence la relation critique ». C'est bien une culture disciplinaire du commentaire $^{4}$ qui se met en place à partir de 1880, à travers la dissertation et l'explication de texte. C'est sur cette dernière «que l'on compte pour faire de la dissertation littéraire la clé de voute du nouvel enseignement du français » (Chervel, 2006, p. 688). De ce point de vue, dissertation et explication sont les deux versants d'un même exercice métatextuel, l'un écrit, l'autre oral ${ }^{5}$. L'explication de texte va alors adopter plusieurs caractéristiques qui vont marquer durablement l'exercice: importance de la méthode, concision, méfiance à l'égard des impressions personnelles.

9 À partir de 1880 , le texte fait l'objet d'une observation à caractère scientifique qui s'inscrit dans la lignée des «leçons de choses» (Jey, 1998, p. 85). Lorsque les IO de 1911 expliquent que l'explication doit « faire valoir les difficultés et après discussion les résoudre » (Mareuil, 1969, p. 35), elles instituent le texte comme problème, et l'exercice comme une réponse organisée à ce problème. On peut donc supposer que la problématique de lecture, que les IO de 1987 revendiquent comme fondatrice de l'exercice, est une héritière de ce mode de lecture des textes. Si l'accompagnement didactique des manuels se renforce à partir des années 1920 (Brunel et al., 2019), c'est afin, non seulement d'accompagner la lecture, mais aussi de la guider et d'orienter l'observation des textes. Dès lors, l'approche méthodologique devient centrale, notamment à travers la question du plan qui parcourt les manuels et les rares copies d'élèves conservées du début du xx siècle (Houdart-Mérot, 1998).

Les programmes s'inscrivent en opposition à l'exercice rhétorique du discours, fondé sur l'amplification (Chervel, 2006). C'est sans doute pourquoi ils ne cessent de caractériser la nouvelle explication à travers sa précision et sa concision. Il faut désormais "éviter la digression qui fait perdre de vue le texte » (IO, 1911; Mareuil, 1969, p. 35). G. Lanson (1903, p. 162) critique le délayage dans l'écriture d'imitation de l'enseignement rhétorique : "Il nous reste à bien concevoir qu'il est plus salubre pour de jeunes esprits d'être exercés à résumer qu'à délayer, à simplifier qu'à amplifier ». Il 
lui reproche aussi sa part d'imagination, d'invention : «Il nous reste peut-être à cesser de louer et de provoquer les jets d'imagination et les constructions logiques, toute une brillante invention qui donne l'habitude de ne pas regarder ce qui est, et de préférer un bel effet à une vérité simple " (ibid.). Les instructions, les manuels, restent d'ailleurs silencieux sur la part d'invention (au sens d'inventio) dans la dissertation de la III ${ }^{\mathrm{e}}$ république (Jey, 1998, p. 113).

11 Dès lors, l'explication de texte, ou de la dissertation doivent se méfier des impressions personnelles. Selon G. Lanson, « la critique impressionniste » est celle de «l'homme qui décrit ce qui se passe en lui quand il lit un livre, sans rien affirmer de plus que ses réactions intérieures » (Charles \& Lanson, 1993, p. 494). Certes, il rejette ici la critique rhétorique qui juge les œuvres en fonction d'un modèle parfait, loue et énumère la beauté des effets, avec éloquence mais sans beaucoup d'exégèse. Mais par là même, il délégitime l'implication du sujet dans la lecture, et souhaite que sa méthode « donne la chasse à cet impressionnisme " (ibid.), qui n'est pas enseignable, pas objectivable, pas méthodique. Enfin, il souligne aussi le paradoxe et la tension que cela génère : « Il nous est à la fois impossible d'éliminer notre réaction personnelle et dangereux de la conserver " (ibid., p. 496). Si la littérature devient un objet autonome à l'égard de la rhétorique, elle est désormais dépendante de la science.

L'exercice de l'explication de texte se caractérise donc par des contraintes inhérentes à une pratique métatextuelle, adossée à une méthode scientifique, qui privilégie une lecture de commentaire. Ces contraintes contribuent à l'autonomisation de l'exerciceC'est ce qu'affirment les IO de de 1890 et de 1909 : «le centre de gravité des études secondaires doit être dans l'explication"; "la lecture expliquée, trop subordonnée à l'histoire littéraire, a passé du second plan au premier et devient de plus en plus, dans sa précision nouvelle, un instrument d'éducation intellectuelle et morale " (Jey, 1998, p. 82, 84). Ces instructions sont très claires : ce n'est pas l'histoire littéraire qui est un objet à apprendre, mais l'exercice en lui-même. Or cette autonomisation est sans doute un premier pas vers une réification de l'exercice, ce qui génère plusieurs tensions au sein des instructions officielles elles-mêmes.

\section{Des Instructions en tensions, un exercice réifié : 1880-2001}

Si la discipline française procède par sédimentations (Schneuwly, 2007), plus que par ruptures (Denizot, 2014), les IO insistent pourtant sur ces dernières et renouvèlent les exercices sans dire ce qu'ils doivent aux précédents. En effet, «la réification et la naturalisation d'un exercice en genre interdit de voir la solidarité avec d'autres exercices qui appartiendraient pourtant, dans une vision plus large, au même genre " (Daunay, 2005, p. 54). Ainsi nombre d'études ont-elles montré que la dissertation se sédimente avec l'exercice du discours, jusqu'aux années 1920 (Chervel, 2006 ; Jey, 1998 ; Houdart-Merot, 1998). Qu'en est-il de l'explication de texte? 


\section{Sédimentation des pratiques de l'enseignement rhétorique avec l'explication de texte} cause de la fonction morale de la littérature enseignée, selon la devise multum, non multa (car « il s'agit moins de lire que de relire», Chervel, 2006, p. 481) mais aussi parce qu'il répond aux exigences d'une pratique lectorale de commentaire (Belhadjin, Perret, 2019). La principale modification est que l'extrait, pour répondre aux exigences méthodologiques, doit désormais être bref. Les IO de 1911 recommandent de «choisir un texte intéressant, d'étendue mesurée, qui forme cependant un tout » (Mareuil, 1969, p. 35) et l'extrait de 15 à 20 lignes s'institutionnalise avec la réforme de l'Épreuve Anticipée de Français de 1969 (Denizot, 2014). La critique de l'extrait isolé qui voit le jour dans les années 1980 conteste bien plus le type d'ancrage que l'objet lui-même (Denizot, 2019, Louichon \& Eugène, 2019). Si l'extrait demeure, que ce soit sous forme de morceaux choisis, dans le cadre de l'œuvre intégrale, ou du groupement de textes, c'est parce que, dans l'enseignement secondaire, on ne peut observer, de façon concise et méthodique, un extrait long et encore moins une œuvre intégrale.

L'explication de texte de 1880 conserve aussi l'approche grammaticale de l'explication latine, notamment parce que l'étude de la langue est considérée comme scientifique (elle occupe de plus en plus de place dans les épreuves de l'agrégation pour les mêmes raisons; Bompaire-Evesque, 2002). Dans l'enseignement rhétorique: "Expliquer un texte latin, c'est le faire passer en français en justifiant les points délicats de la traduction ou encore en révisant, à l'occasion du texte, les règles principales de morphologie, de syntaxe, de prosodie, de poétique ou de rhétorique que le professeur a dogmatiquement enseignées" (Chervel, 1985, p. 8). On explique tout d'abord les auteurs latins car on n'a pas à expliquer un auteur français dont on connait la langue. C'est en 1840 (Jey, 1998) que l'exercice évolue: les méconnaissances concernant la langue et la littérature françaises classiques entrainent des contresens en thème latin (Houdart-Merot, 1998). L'explication d'auteurs français se met alors en place. H. Fortoul, en 1852, en rappelle les règles qui annoncent l'exercice de 1880 (Jey, 1998, p. 80).

Le professeur doit donc attacher une très grande importance à l'explication des auteurs français; il faut qu'il détermine la valeur des mots et la propriété des termes, leurs rapports et leurs acceptions diverses; qu'il rende sensible la liaison des idées, qu'il distingue les idées principales et les idées accessoires; qu'il montre dans quel ordre elles sont disposées [...].

L'explication de 1880 continue dès lors d'accorder une grande importance à la grammaire, au lexique, à la syntaxe, comme à l'époque où elle servait à la traduction latine (Massol, 2004).

Si le format de l'extrait et l'étude de la grammaire sont conservés, c'est parce qu'ils demeurent en cohérence avec la lecture métatextuelle. En revanche, le maintien de la fonction morale de la littérature, présente dans l'enseignement rhétorique, crée des tensions, comme le souligne M. Jey (1998, p. 85). En 1890, l'exercice est défini comme « une leçon de choses morale professée par des écrivains de génie » (ibid.). Il s'agit alors 
à la fois de célébrer les auteurs et d'observer scientifiquement un texte, ce qui semble assez paradoxal. Commenter et admirer deviennent alors synonymes et visent tout autant à former le gout que le raisonnement, même s'il s'agit désormais de célébrer la belle page, non d'en écrire une. L'exercice consiste en réalité à admirer la langue du texte, à retrouver l'idée qui la sous-tend et à en dégager l'architecture, tout aussi admirable, au moins jusqu'aux années 1970. Ainsi les IO de 1977 continuent-ils de mêler l'objectivité supposée de l'histoire littéraire et la formation morale : "Les maîtres ne négligeront pas les œuvres qui tout en offrant un intérêt largement humain, constituent en même temps des témoignages sur les conditions matérielles, économiques et sociales d'une civilisation » (Ministère de l'Éducation nationale, 1977, p. 63).

19 Les sédiments ne se déposent pas en strates régulières, certains sont en harmonie avec le nouvel exercice, d'autres moins. Si la réification d'un exercice interdit de voir ce qu'il doit au précédent, elle a aussi d'autres conséquences : les programmes déplorent des pratiques pourtant générées par l'exercice lui-même, et reprochent aux programmes antérieurs d'en être responsables.

\section{Tensions et dérives de l'exercice}

Les programmes, alors qu'ils demandent aux élèves d'adopter une posture de commentateur objectif, ne cessent, dans le même temps, de regretter la pratique d'une lecture standardisée, mécanique, qui fait «perdre de vue le principal, c'est-à-dire le texte » (IO, 1890 ; Jey, 1998, p. 83). Les IO caractérisent en effet l'explication par ce qu'elle ne doit pas être, en soulignent les dérives, tout au long du corpus, quelles que soient les méthodes et leurs évolutions. La continuité de l'exercice repose ainsi sur des contraintes et des paradoxes qui se sédimentent depuis l'origine, et entrainent des malentendus didactiques, sources de tensions. Le discours officiel sur la paraphrase a, par exemple, été étudié par B. Daunay (1997, 1999b, 2002). En 1909 les programmes demandent d'« éviter [...] la paraphrase qui l'énerve [le texte]» (Mareuil, 1969, p. 35) ceux de 1987 affirment encore que la lecture méthodique "rejette la paraphrase » (Ministère de l'Éducation nationale, 1987, p. 9). Ce rejet est paradoxal, puisque la paraphrase (en tant que reformulation) est nécessaire à l'élaboration de tout commentaire de texte (Daunay, 2004). Les déplorations sur l'élaboration du plan sont aussi fréquentes. En 1909, les programment rappellent qu'il faut: "Éviter [...] les divisions faussement méthodiques qui, d'une page vivante, font une stérile anatomie " (Mareuil, 1969, p. 35). En 1987, la lecture méthodique «ne mime pas, passivement, le développement linéaire du texte » (Ministère de l'Éducation nationale, 1987, p. 9). Ces reproches sur la «passivité », la «stérilité » des lectures peuvent être compris comme un manque d'implication de l'élève, dont l'exercice se méfie pourtant.

21 L'exercice s'étant réifié, les IO n'en interrogent pas les fondements mais expliquent ces dérives par une mauvaise définition de l'exercice dans les IO précédentes. En effet, « la délimitation $\mathrm{d}^{\prime}[\mathrm{un}$ ] exercice par son opposition à d'autres [est] essentielle dans sa caractérisation » (Daunay, 2005, p. 49). La lecture méthodique de 1987 invalide ainsi l'exercice de l'explication: "Les exigences d'une lecture méthodique permettent de donner plus de rigueur et plus de force à ce que l'on nomme d'habitude explication » (Ministère de l'Éducation nationale, 1987, p.9). L'exigence d'un supplément de « rigueur » et de «force » souligne l'importance de la méthode, et passe en réalité par 
un renforcement des savoirs déclaratifs sur la langue des textes puisque dans la lecture méthodique "la compréhension du texte est réduite à la maitrise de connaissances linguistiques et sémiotiques » (Petitjean, 2014, p. 31). La lecture analytique est mise en place pour lutter contre cette dérive, les IO soulignant que les « outils d'analyse » ne sont pas «une fin en soi» et rejetant explicitement le formalisme de la lecture méthodique: "Les précédents programmes insistaient sur les méthodes: ce souci légitime induisait en pratique bien des formalismes et des technicismes » (Ministère de l'Éducation nationale, 2001, p. 8). Mais l'exercice n'y échappe pas: s'il consiste à "développer la capacité d'analyses critiques et autonomes » (ibid.), il s'agit bien plus d'analyser le texte que ses impressions de lecture. L'exercice se renouvèle donc plusieurs fois mais son essence demeure métatextuelle. Les noms qu'il adopte à partir des années 1980 mettent d'ailleurs en valeur l'importance de la méthode (lecture méthodique) et de l'analyse (lecture analytique).

Ainsi les dérives sont-elles identifiées comme émanant des élèves (qui paraphrasent, proposent des plans inadéquats), des professeurs (qui continuent de transmettre des pratiques dépassées, faisant de l'explication de texte alors qu'elle devrait être méthodique, de la lecture méthodique alors qu'elle devrait être analytique), ou des dispositifs énonciatifs des IO elles-mêmes (Michel, 1999). Elles révèlent surtout que la lecture d'un texte littéraire à l'école consiste à le transformer en problème, puis en problématique, en appliquant une méthode afin de trouver un plan pour commenter un extrait, forcément admirable.

Cette naturalisation de l'exercice en genre du discours métatextuel, qui se combine avec l'approche méthodico-scientifique des textes, a aussi des conséquences, à partir des années 1980, sur les théories de référence qui sous-tendent l'exercice. Il existe en effet une "hiérarchie des formes de lectures " (Daunay, 1999a, p. 30), lisible dans les programmes qui se succèdent et cette hiérarchie dévalorise certains rapports aux textes (ibid.). Dans une explication de texte fondée sur l'approche méthodologicoscientifique, certaines théories considérées comme légitimes parce que scientifiques à une époque donnée ne le sont plus ensuite. Par ailleurs, l'arrivée des théories du lecteur met à mal l'approche métatextuelle.

\section{Reconfigurations des approches des textes littéraires : 1983-2019}

La nouvelle configuration disciplinaire qui se met en place dans les années 1980 s'accompagne d'un élargissement des corpus et de modifications des exercices. Elle est aussi concomitante à la création de la didactique du français et aux théories de P. Bourdieu en sociologie de l'éducation qui mettent en garde contre certaines formes de lectures cultivées introduisant des effets de connivence et de distinction. Il s'agit alors de renouveler les savoirs objectivables, à une époque où l'accès au baccalauréat se démocratise (ou se massifie). Tous ces éléments vont entrainer une reconfiguration de l'étude de la langue et de l'histoire littéraire scolaire, à des degrés divers, et l'apparition de la question du sujet lecteur qui peine à se légitimer. 


\section{De la grammaire aux sciences du langage} dans les années 1970-1980, lorsque se créent les sciences du langage, au profit des «théories immanentes des textes (linguistique structurale puis textuelle, sémiotique des textes, nouvelle critique)»(Petitjean, 2014, p.13). Les travaux issus de la linguistique et de la théorie de la communication sont alors au cœur des débats aussi bien dans l'enseignement secondaire que primaire (Bishop, 2008). Si le texte officiel de 1987 rappelle qu'il faudra étudier la grammaire, la syntaxe, le lexique, pratiques issues de la traduction latine, s'y ajoutent la morphologie, l'énoncé et l'énonciation, les structures apparentes et profondes (Ministère de l'Éducation nationale, 1987, p. 9). L'approche se modifie et devient une étude des «principales opérations constitutives du discours » (Ministère de l'Éducation nationale, 1983), de la "communication et de l'expression » (Ministère de l'Éducation nationale, 1987, p. 7).

Il s'agit bien ici de renforcer la scientificité de l'exercice, avec l'idée qu'une approche linguistique outillée permettra de lutter contre les inégalités scolaires.

L'accent [est] mis sur les indices formels susceptibles d'étayer les interprétations et qui ont l'avantage de fournir à la discipline des savoirs objectivables et des exercices évaluables, procurant, de ce fait, un regain de légitimité aux études de lettres par rapport aux disciplines scientifiques (Petitjean, 2014, p. 18).

La lecture méthodique relève donc d'une "problématique logico-grammaticale" (Michel, 1999, p. 83), le texte littéraire devient « un objet linguistique, qui doit faire l'objet d'une étude immanente» (Petitjean, 2014, p. 16), non d'une lecture contextualisante, caractéristique de l'histoire littéraire. La hiérarchie est alors reconfigurée, l'histoire littéraire auparavant fondatrice de l'exercice, est minorée au profit des théories du texte (Michel, 1998).

\section{Une histoire littéraire reconfigurée}

Si l'histoire littéraire est considérée comme une science à la fin du xixe siècle, notamment parce que la démarche historique permet la mise en place de savoirs déclaratifs (biographie, contexte historique de rédaction...) qui se déclinent en exercices, elle devient sujette à débats à partir des années 1960. On lui reproche justement son manque de scientificité : son dogmatisme, son absence de méthode, son canon, ses présupposés idéologiques et ses savoirs non interrogés (Daunay, 2007a). Ainsi, S. Delessalle, membre du " groupe Chevalier " qui réfléchissait à la réforme de l'enseignement littéraire au lycée au début des années 1970 (Delcambre, 1999), reproche à l'explication de textes d'être fondée sur la connivence culturelle et « la célébration des valeurs admises de l'histoire littéraire » (Delesalle, 1970, p. 90). Cela va avoir pour effet de modifier l'approche scolaire de cette dernière et la place qu'elle occupe dans l'exercice.

À partir des années 1980, l'histoire littéraire scolaire est clairement contestée dans les programmes. Tout d'abord, elle n'est plus nommée de façon explicite car elle n'est plus la théorie de référence majeure. Aucun nom ne la désigne dans les IO de 1982. En 1987, elle est renommée «l'éclairage historique " (Ministère de l'Éducation nationale, 1987, p. 8), et elle apparait, non pas dans la définition de l'exercice en lui-même, mais dans les "perspectives de lecture»(ibid.). Ensuite, certaines notions, qui la fondaient 
disparaissent, notamment la biographie de l'auteur, ou bien ne lui appartiennent plus en propre, comme le genre littéraire, qui peut désormais être étudié sans référence à son histoire (Perret, 2019). La lecture méthodique en est le reflet : elle «n'attribue pas à l'auteur, a priori, une intention [...], ne s'enferme pas dans des préjugés esthétiques » (Ministère de l'Éducation nationale, 1987, p. 9). Enfin, elle est renouvelée : elle intègre des éléments de l'histoire culturelle, de la sociologie de la littérature. Les IO soulignent "l'importance des rapports entre une production littéraire et son environnement social, économique et politique " (ibid., 1982, p. 50) ou encore la nécessité d'étudier «l'environnement humain et culturel, voire la vie quotidienne [...] les circonstances économiques et politiques, sans négliger les codes et conventions littéraires de l'époque » (ibid., 1987, p. 8). En 2001, elle devient « L'histoire littéraire et culturelle ».

Une fois de plus, ces IO de 2001 considèrent que si l'histoire littéraire est difficile à enseigner, c'est à cause d'une approche contestable de la chronologie dans les IO de 1987 :

Les précédents programmes indiquaient comme cadre les siècles de l'histoire de la France. Cadre large et généreux mais si vague qu'il présentait deux inconvénients : l'un d'ordre pratique, car quelques aspects seulement étaient traités, l'autre d'ordre déontologique car il supposait une certitude établie sur l'histoire et son déroulement, donc une vision idéologiquement contrainte et contraignante, même si elle n'était pas avouée ni même pensée comme telle (ibid., p. 9).

Pourtant, ces IO continuent de préconiser un enseignement qui «permet de saisir les grandes scansions historiques que constituent les changements majeurs dans les façons de penser et de sentir, mais aussi dans les façons de s'exprimer » (ibid.)

L'histoire littéraire est en réalité réduite à deux aspects, la contextualisation et la périodisation (qui est pourtant aussi remise en question dans les recherches des années 1960, Béhar \& Fayolle, 1990). Les IO de 1982 à 2019 ne cessent de rappeler la nécessité de doter les élèves "de solides repères chronologiques [...] [en] situant [l'œuvre] dans son environnement et dans l'histoire » (1982, p. 47) ou encore de leur «redonner [...] le sens de la continuité historique [...]. Le professeur propose une histoire littéraire chronologiquement organisée, sans simplification abusive des découpages » $(1987$, p. 8$)$.

La hiérarchisation se fait donc au détriment de l'histoire littéraire qui se maintient au prix d'une réduction de ses contenus et est le plus souvent cantonnée à l'introduction de l'exercice, en quelque sorte, une " périphérisation ".

34 Si la hiérarchie des modes de lectures est modifiée, l'exercice conserve toutefois ses deux théories fondatrices. Lire un texte, c'est le commenter en présentant, brièvement, son contexte de rédaction et en étudiant, longuement, sa langue. Mais lorsque les IO affirment aussi que le sens d'un texte est construit par le lecteur, de nouvelles tensions apparaissent.

\section{Illégitimité des théories du sujet lecteur}

Les théories du sujet lecteur se sont constituées d'abord en rupture avec une pratique herméneutique stricte et une objectivation des lectures. Elles ont donc été investies dans des espaces de lecture concurrents à l'explication de texte et ses avatars. Pourtant, les programmes vont, progressivement, tenter d'articuler droits du texte (au cœur des IO jusqu'en 1987) et droits du lecteur, sans souligner, une fois de plus, les tensions, 
voire les incompatibilités qui existent entre ces deux approches. Depuis les années 1980, l'exercice doit ainsi articuler l'objectivité supposée du savoir apporté par la linguistique et une histoire littéraire reconfigurée avec la subjectivité à l'œuvre dans l'acte de lecture et la pratique interprétative.

La prise en compte de l'élève en tant que lecteur apparait, prudemment, dans les IO de 1987. La lecture méthodique "permet aux élèves d'élucider, de conformer ou de corriger leurs premières réactions de lecteur " (Ministère de l'Éducation nationale, 1987, p. 9). La formule souligne les tensions, même si elles sont occultées : les droits du texte priment sur ceux du lecteur. Si l'élève n'a pas proposé d'emblée une analyse conforme, il doit la corriger. Le passage de la lecture méthodique à la lecture analytique signale le glissement d'une théorie de l'effet vers une théorie de la réception (Langlade \& Rouxel, 2004) en accordant une place plus significative au lecteur dans l'élaboration du sens. En effet, c'est avec la lecture analytique que le terme "interprétation » apparait: "elle a pour but la construction détaillée de la signification d'un texte et constitue donc un travail d'interprétation " (Ministère de l'Éducation nationale, 2002, p.7). Là encore, la tension est lisible. Non seulement le terme interprétation n'est jamais clairement défini (Ahr, 2019), mais la formule "construction détaillée de la signification du texte » souligne que le droit du texte continue de primer, ainsi que les méthodes instituées par l'explication. La formule des programmes de 2006 est du même ordre: "L'objectif de la lecture analytique est la construction et la formulation d'une interprétation fondée» (Ministère de l'Éducation nationale, 2006). On peut comprendre : fondée sur les droits du texte, non du lecteur.

Toutefois on peut noter une timide évolution des positions officielles. Si les dérives technicistes de la lecture méthodique sont essentiellement soulignées par les premiers didacticiens de la littérature (Michel, 1998, Langlade, 2001), celles de la lecture analytique existent non seulement dans ce champ de recherche (par exemple, De Beaudrap 2006, Lacelle \& Langlade, 2007) mais aussi lors de séminaires, conférences du ministère de l'éducation nationale (Laudet, 2011 ; Vibert, 2011).

Si la lecture analytique est un exercice en tensions, c'est donc pour deux raisons : d'une part, elle est le produit de trois conceptions de ce qui fait la littérarité d'un texte, d'une œuvre, de ce qu'est la littérature; d'autre part, la réification de l'exercice génère inévitablement une hiérarchisation de ces modalités de lecture, pourtant présentées dans les IO comme devant exister sur le même plan. Si deux d'entre elles (histoire littéraire, langue) trouvent leur unité dans leur dimension métatextuelle, la théorie du sujet lecteur a plus de mal à se légitimer. En effet, ni le sujet lecteur ni le sujet scripteur ne peuvent occuper une place importante dans une conception métatextuelle de l'étude de la littérature. Ainsi la lecture participative est-elle hiérarchisée au profit de la lecture distanciée, rendant difficile le continuum entre ces deux postures de lecteur (Daunay \& Dufays, 2016). Ce ne sont pas tant les outils qui ne permettent pas l'interprétation que la conception réifiée de la lecture métatextuelle. En effet, la métatextualité "n'est jamais en principe de l'ordre de la fiction narrative ou dramatique [...] Le métatexte, lui, est non fictionnel par essence.» (Genette, 1982, p. 554). C'est donc l'«activité fictionnalisante» (Langlade, 2007) des lecteurs et des scripteurs qui est contestée dans l'approche métatextuelle. On peut dès lors interroger, pour finir, les nouveaux programmes, au regard de ces analyses. 


\section{Place du métatexte dans les programmes de français actuels}

En 2019, l'exercice retrouve la dénomination originale d'explication de texte, mais il est un avatar de la lecture méthodique dont il conserve la théorie de l'effet : «l'explication de texte fonde son interprétation sur une analyse précise des principaux effets de sens» (Ministères de l'Éducation nationale, 2019). En réalité, au-delà de ces dénominations, l'exercice trouve son unité dans sa dimension métatextuelle, il correspond à un genre scolaire stabilisé, naturalisé, réifié, reposant sur une observation méthodique des textes. Ainsi, lorsque les programmes de 2019 écrivent que «la méthode est laissée au choix du professeur », on peut comprendre: qu'importe la méthode, pourvu qu'on ait du métatexte; voire, nous savons que les professeurs proposeront, toujours, une lecture métatextuelle, tant cette approche s'est réifiée dans la culture disciplinaire. Adopter la dénomination que l'exercice avait sous la ${ }_{\text {III }}^{\mathrm{e}}$ république rappelle aussi, d'une part, que cette période est encore considérée, à tort, comme un âge d'or de l'école et refuse, d'autre part, à l'exercice la spécificité scolaire qu'il avait acquise en devenant lecture méthodique ou analytique, le terme «explication de texte " n'ayant jamais disparu des épreuves de l'agrégation et du Capes.

40 Les programmes de 2019 suppriment aussi l'écriture d'invention. Créé en 2001, l'exercice était une tentative de remise en question de la dimension lectorale de la dissertation, et fit l'objet de violentes polémiques (Daunay \& Denizot, 2018). Il s'agissait en effet d'un exercice de nature hypertextuelle ${ }^{6}$ qui remettait en question l'écriture métatextuelle, heurtant ainsi la culture disciplinaire. Les premières recherches en didactique sur l'écriture d'invention ont montré sa contamination par la dissertation, l'exercice conservant une dimension métatextuelle, certes atténuée (Daunay, 2003, Petitjean, 2003). Mais, depuis 2008, l'exercice évoluait vers «une certaine autonomisation [...] perd[ant] peu à peu ses ancrages initiaux dans les autres exercices institués» (Daunay \& Denizot, 2018, p. 167) et devenant plus hypertextuel. Sa suppression est bien le signe que l'approche métatextuelle demeure au cœur de l'institution et des pratiques, les oppositions à sa disparition ayant été quasi inexistantes dans le corps enseignant.

41 Cette approche métatextuelle est si ancrée dans la discipline que ses enjeux gagnent l'école primaire. Les programmes de 2015 parlaient « d'appropriation subjective des œuvres » (Ministère de l'Éducation nationale, 2015, p. 108) et de "posture d'auteur » (ibid., p. 11). Ces termes ont disparu dans les réajustements de 2018, au profit de nouveaux enseignements : apporter les «premiers éléments de contextualisation dans l'histoire littéraire » (ibid., 2018, p. 25) et «identifier les différents genres représentés et repérer leurs caractéristiques majeures " (ibid.). L'approche métatextuelle gagne donc du terrain, dans les IO tout du moins.

Dans les programmes, la lecture analytique est donc bien un avatar de la lecture méthodique qui a elle-même conservé certaines caractéristiques de l'explication de texte, elle-même redevable de l'exercice de l'explication latine. Contrairement au commentaire qui, sous une même dénomination, génère différents exercices au fil de son histoire et de ses prescriptions officielles (Daunay, 2005), l'explication de texte, au- 
delà de ses différentes dénominations, répond à « des codes et des règles qui dans le fond ont peu évolué » (Denizot, 2014, p. 240) depuis l'origine, ce qui en fait un « véritable genre scolaire » (ibid.). Elle trouve en réalité son unité dans la pratique du commentaire, de faits de langue, du contexte historique de rédaction. De plus, cette pratique permet, une évaluation qui se veut objective, enjeu important pour un exercice devenu une épreuve discriminante au baccalauréat.

Si l'exercice est en tension, c'est sans doute parce que la dimension métatextuelle qui en est l'essence, s'est réifiée, naturalisée. Les instructions officielles, depuis l'origine, prônent une finalité esthétique, politique, morale à l'enseignement de la littérature, et demandent aussi désormais une activité interprétative de sujet lecteur. Mais la réification de l'exercice amène à occulter la dimension politique de la littérature, à édulcorer l'expérience esthétique, à minorer l'implication du lecteur, au profit du commentaire d'une œuvre forcément objectivée dans la lecture métatextuelle. Au lycée, contrairement aux finalités affichées, on ne lit en réalité pas, pour mieux comprendre le monde et se connaitre soi-même, mais pour répondre aux contraintes d'un exercice qui s'est autonomisé dès l'origine.

Prendre en compte le sujet lecteur/scripteur dans l'explication de texte et ses avatars supposerait donc de bouleverser les hiérarchies des modalités de lecture. Mais le risque, cette fois-ci, serait de réintroduire les effets de connivence et de distinction reprochés à l'histoire littéraire, au bénéfice "d'un empirisme spontanéiste, qui négligerait la nécessité didactique de la construction d'outils conceptuels " (Daunay, 2007b p. 47). Si « d'un point de vue didactique, la littérature est affaire de savoirs et de savoir-faire objectivement descriptibles et enseignables" (ibid. p. 49), alors, "la normalisation des comportements de lecture est un objectif scolaire incontournable (ibid.). Il s'agirait donc de normaliser les modalités de lecture, pour pouvoir les enseigner, sans pour autant les réifier. L'articulation du sujet lecteur avec une pratique analytique demeure donc non seulement discutée, mais problématique.

\section{BIBLIOGRAPHIE}

AHR, S. (2019). « L'interprétation : un concept stabilisé ? À stabiliser ? ». In : Denizot, N., Dufays, J.L. \& Louichon, B. (dirs). Approches didactiques de la littérature. Namur : presses universitaires de Namur, p. 89-106.

BÉHAR, H. \& FAYOLLE, R. (dirs) (1990). L’Histoire littéraire aujourd'hui. Paris : A. Colin.

BELHADJIN, A. \& PERRET, L. (dirs). (2019). L'Extrait et la fabrique de la littérature scolaire. Bruxelles :

P. Lang.

BISHOP, M.-F. (2008). « Une réforme complexe et polémique : la rénovation du Français à l'école

élémentaire de 1963 à 1972 ». Le Télémaque 34, p. 59-72. En ligne : https://www.cairn.info/journalle-telemaque-2008-2-page-59.htm\#. 
BISHOP, M.-F. (2017). « Une question de méthode : l'approche historico-didactique en français ». In : Dias-Chiaruttini, A. \& Cohen-Azria, C. (dirs). Théories-didactiques de la lecture et de l'écriture. Villeneuve d'Ascq : Presses universitaires du Septentrion, p. 225-239.

BOMPAIRE-EVESQUE, C. (2002). « Le procès de la rhétorique dans l'enseignement supérieur français à la fin du XIX ${ }^{\mathrm{e}}$ siècle ». Revue d'histoire littéraire de la France 102 (3), p. 389-404. En ligne : https:// www.cairn.info/revue-d-histoire-litteraire-de-la-france-2002-3-page-389.htm.

BRUNEL, M. et al. (2019). « D'un siècle l'autre (1900-2000) : que nous révèlent les avatars de l'extrait ? Une comparaison France-Belgique-Québec ». IN : Belhadjin, A. \& Perret, L. (dirs). L'Extrait et la fabrique de la littérature scolaire. Bruxelles : P. Lang, p. 77-94. En ligne : https:// dial.uclouvain.be/pr/boreal/object/boreal:220835.

CHARLES, M. (1985). L'Arbre et la source. Paris : Éditions du Seuil.

CHARLES, M. \& LANSON, G. (1993). « Amateurs, savants et professeurs ». Poétique 96, p. 493-508.

CHERVEL, A. (1985). « Sur l'origine de l'enseignement du français dans le secondaire ». Histoire de l'éducation 25, p. 2-10. En ligne : https://www.persee.fr/doc/ hedu_0221-6280_1985_num_25_1_1283.

CHERVEL, A. (2006). Histoire de l'enseignement du français du XVII au XXe siècle. Paris : Retz.

DAUNAY, B. (1997). «La paraphrase dans le commentaire de texte littéraire ». Pratiques 95, p. 97-115. En ligne : https://www.persee.fr/doc/prati_0338-2389_1997_num_95_1_1814.

DAUNAY, B. (1999a). «La lecture littéraire : les risques d'une mystification ». Recherches 30, p. 29-59. En ligne : https://revue-recherches.fr/wp-content/uploads/2016/01/

R30_29-59_Daunay.pdf.

DAUNAY, B. (1999b). « La paraphrase dans les instructions officielles depuis un siècle ». In : Petitjean, A. \& Privat, J.-M. (dirs). Histoire de l'enseignement du français et textes officiels. Actes du colloque de Metz. Metz : Université de Metz, p. 193-214.

DAUNAY, B. (2002). La Paraphrase dans l'enseignement du français. Bruxelles : P. Lang.

DAUNAY, B. (2003). « Les liens entre écriture d'invention et écriture métatextuelle dans l'histoire de la discipline. Quelques interrogations ». Enjeux. Revue de formation continuée et de didactique du français 57, p. 9-24. En ligne : https://www.archives-ouvertes.fr/hal-01354208/document.

DAUNAY, B. (2004). « Réécriture et paraphrase. Contribution à une histoire des pratiques d'écriture scolaire ». Le français aujourd'hui 144 (1), p. 25-32. En ligne : https://www.cairn.info/ revue-le-francais-aujourd-hui-2004-1-page-25.htm.

DAUNAY, B. (2005). « Le commentaire : exercice, genre, activité ? ». Les Cahiers Théodile 5, p. 49-61. En ligne : http://f.tourbez.free.fr/Theodile/Programme/Theodile/Download/Tires_a_part/ Daunay_Cahiers_Theodile_5.pdf.

DAUNAY, B. (2007a). «État des recherches en didactique de la littérature ». Revue française de pédagogie 159, p. 139-189. En ligne : http://ife.ens-lyon.fr/publications/edition-electronique/ revue-francaise-de-pedagogie/INRP_RF159_12.pdf.

DAUNAY, B. (2007b). « Le sujet lecteur : une question pour la didactique du français ». Le français aujourd'hui 157 (2), p. 43-51. En ligne : https://www.cairn.info/revue-le-francais-aujourdhui-2007-2-page-43.htm. 
DAUNAY, B. (2019) «Le genre de la "lettre métatextuelle" ». In : Denizot, N. \& Ronveaux, C. (dirs). La Lettre enseignée. Perspectives historiques et européennes. Grenoble : Université Grenoble Alpes éditions, p. 61-72. En ligne : https://books.openedition.org/ugaeditions/9986?lang=fr.

DAUNAY, B. \& DENIZOT, N. (2018). «L'écriture d'invention : une réforme française et des recherches didactiques francophones ». In : Aeby Daghé, S. \& Guernier, M.-C. (dirs). Contextes institutionnels, réformes et recherches en didactique du français. Namur: Presses universitaires de Namur, p. $157-180$.

DAUNAY, B. \& DUFAYS, J.-L. (2016). « La lecture littéraire en débat, pour en finir ? ». In : Ronveaux C. (dir.). Enseigner les littératures dans le souci de la langue. Bruxelles: P. Lang, p. 211-232.

DE BEAUDRAP, A.-R. (2006). « Quand les humanités doivent résister aux méthodes ». In : Chabanne, J.-C. (dir.). Parler, lire, écrire dans la classe de littérature : l'activité de l'élève/le travail de l'enseignant/la place de l'œuvre. Actes de conférence en CD-ROM. $7^{\mathrm{e}}$ Rencontres de didactique de la littérature. Montpellier, avril 2006.

DELCAMBRE, I. (1999). «L'oral au collège, émergence d'une spécificité ». Pratiques 101-102, p. 59-76. En ligne : https://www.persee.fr/doc/prati_0338-2389_1999_num_101_1_1832.

DELESALLE, S. (1970). « L'explication de textes, fonctionnement et fonction ». Langue française 7, p. 87-95. En ligne : https://www.persee.fr/doc/lfr_0023-8368_1970_num_7_1_5509.

DELFAU, G. (1978). « Le positivisme, l'histoire de la critique et nous ». Romantisme 21-22, p. 233-238. En ligne : https://www.persee.fr/doc/roman_0048-8593_1978_num_8_21_5221.

DENIZOT, N. (2013a). « La dissertation : un genre scolaire argumentatif ? Perspective historique ». Pratiques 157-158, p. 165-176. En ligne : https://journals.openedition.org/pratiques/3823.

DENIZOT, N. (2013b). La Scolarisation des genres littéraires (1880-2010). Bruxelles : P. Lang.

DENIZOT, N. (2014), «L'enseignement du français au lycée : mutation ou reconfiguration ? ». In :

Meskel-Cresta, M. et al. (dirs). École et mutation. Reconfigurations, résistances, émergences. Louvain-laNeuve : De Boeck, p. 231-241. En ligne : https://www.cairn.info/ecole-etmutation--9782804184742-page-231.htm.

DENIzOT, N. (2019). « Qu'est-ce qu'un extrait ». IN : Belhadjin A. \& Perret L. (dirs.) L'Extrait ou comment se scolarise la littérature. Bruxelles : Peter Lang, p. 25-38.

DUFAYS, J.-L. (2016). « La lecture littéraire, histoire et avatars d'un modèle didactique ». Tréma. Revue internationale en science de l'éducation et didactique 45, p. 9-17. En ligne : https:// journals.openedition.org/trema/3486.

GENETTE, G. (1982). Palimpsestes. La littérature au second degré. Paris : Éditions du Seuil.

HOUDART-MEROT, V. (1998). La Culture littéraire au lycée depuis 1880. Rennes : Presses universitaires de Rennes.

JEY, M. (1998). La Littérature au lycée. L'invention d'une discipline, 1880-1925. Metz : Université de Metz.

LACELLE, N. \& LANGLADE, G. (2007). « Former des lecteurs/spectateurs par la lecture subjective des œuvres ». In Dufays, J.-L. (éd.). Enseigner et apprendre la littérature aujourd'hui, pour quoi faire? Sens, utilité, évaluation. Louvain-la-Neuve : Presses universitaires de Louvain, p. 55-64.

LANGLADE, G. (2001). «Et le sujet lecteur dans tout ça? ». Enjeux. Revue de formation continuée et de didactique du français 51-52, p. 53-62. 
LANGLADE, G. (2007). « La lecture subjective ». Québec français 145, p. 71-73. En ligne : https:// www.erudit.org/fr/revues/qf/2007-n145-qf1178006/47315ac/.

LANGLADE, G. (2014). « La lecture subjective est-elle soluble dans l'enseignement de la littérature? ». Études de lettres 1, p. 47-63. En ligne : https://journals.openedition.org/edl/608.

LANGLADE, G. \& ROUXEL, A. (dirs) (2004). Le Sujet lecteur. Lecture subjective et enseignement de la littérature. Rennes : Presses universitaires de Rennes.

LANSON, G. (1903). « Les études modernes dans l'enseignement secondaire ». In : Lavisse, E., L'Éducation de la démocratie. Leçons professées à l'École des hautes études sociales, Paris : F. Alcan, p. $162-163$.

LANson, G. (1965) [1925]. « La Méthode de l'histoire littéraire ». In : Peyre, H. (éd.). Essais de méthode, de critique et d'histoire littéraire. Paris : Hachette, p. 30-35.

LAUDET, P. (2011). « Explication de texte littéraire : un exercice à revivifier. Intervention de Patrick Laudet, inspecteur général de l'Éducation nationale, groupe des lettres, en séminaire national ». Ressources pour le lycée général et technologique, juin 2011. Paris : Ministère de l'Éducation nationale, de la Jeunesse et de la Vie associative/Direction générale de l'enseignement scolaire. En ligne : https://media.eduscol.education.fr/file/Francais/09/5/ LyceeGT_Ressources_Francais_Explication_Laudet_182095.pdf.

LOUICHON, B. \& EUGÈNE, M. (2019). «L'envers de l'extrait : relations didactiques entre extraits et œuvres en France de 1880 à nos jours du primaire au secondaire ». IN : Belhadjin A. \& Perret L. (dirs). L'Extrait et la fabrique de la littérature scolaire. Bruxelles : Peter Lang, p. 55-74.

MAREUIL, A. (1969). « Les programmes de français dans l'enseignement du second degré depuis un siècle (1872-1967) ». Revue française de pédagogie 7, p. 31-45. En ligne : https://www.persee.fr/doc/ rfp_0556-7807_1969_num_7_1_1771.

MASSOL, J.-F. (2004). De l'institution scolaire de la littérature française (1870-1925). Grenoble : Éditions Littéraires et Linguistiques de l'Université de Grenoble.

MICHEL, R. (1998). « Lecture méthodique ou méthode de lecture à l'usage des élèves de lycée. Un objet didactique non identifié ». Pratiques 97-98, p. 59-103. En ligne : https://www.persee.fr/doc/ prati_0338-2389_1998_num_97_1_2481.

MICHEL, R. (1999). « La lecture méthodique à la lumière des instructions officielles : une obscure clarté ». Pratiques 101-102, p. 77-94. En ligne : https://www.persee.fr/doc/ prati_0338-2389_1999_num_101_1_1833.

PERRET, L. (2019). « L'histoire littéraire comme objet d'enseignement : l'apport des Rencontres des chercheurs en didactique de la littérature ». In : Denizot, N., Dufays, J.-L. \& Louichon, B. (dirs). Approches didactiques de la littérature. Namur : presses universitaires de Namur, p. 67-88.

petitjean, A. (2003). « Histoire de l'écriture d'invention au lycée ». Pratiques, 117-118, p. 181-207. En ligne : https://www.persee.fr/doc/prati_0338-2389_2003_num_117_1_2002.

PETITJEAN, A. (2014). « 40 ans d'histoire de la "lecture littéraire" au secondaire à partir de la revue Pratiques ». Pratiques 161-162, p. 1-81. En ligne : https://journals.openedition.org/pratiques/2155. Petitjean, A. \& PRIVAT, J.-M. (1999). « Présentation ». In : Petitjean, A. \& Privat, J.-M. (dirs). Histoire de l'enseignement $d u$ français et textes officiels. Actes du colloque de Metz. Metz : Université de Metz, p. 5-8.

PLISSONNEAU, G., BAZILE, S. \& BOUTEVIN, C. (2017). « La lecture analytique à la fin du collège : un exercice de lecture littéraire ? Points de vue d'enseignants, regards d'élèves ». Repères. Recherches 
en didactique du français langue maternelle 56, p. 91-108. En ligne : https://

journals.openedition.org/reperes/1197.

SCHNEUWLY, B. (2007). «Le Français : une discipline scolaire autonome, ouverte et articulée ». In : Falardeau, E. et al. (dirs). La Didactique du français. Les voies actuelles de la recherche. Québec: Presses de l'université de Laval, p. 9-26.

VIBERT, A. (2011). « Faire place au sujet lecteur en classe : quelles voies pour renouveler les approches de la lecture analytique au collège et au lycée ? Intervention d'Anne Vibert, inspectrice générale, en séminaire national (mars 2011) ». Ressources pour le collège et le lycée. Paris : Ministère de l'Éducation nationale, de la Jeunesse et de la Vie associative/Direction générale de l'enseignement scolaire/Inspection générale de l'Éducation nationale. En ligne : https://eduscol.education.fr/lettres/im_pdflettres/intervention-anne-vibert-lecturevf-20-11-13.pdf.

\section{Corpus de textes officiels}

Ministère de l'Éducation nationale (1977). Bulletin officiel nº 11 du 24 mars 1977.

Ministère de l'Éducation nationale (1982). Français langues anciennes. Classe de seconde, première, terminale. Brochure du CNDP $n^{\circ}$ 6011. Paris : Centre national de documentation pédagogique.

Ministère de l'Éducation nationale (1983). Bulletin officiel no 28 du 17 juillet 1983.

Ministère de l'Éducation nationale (1987). Bulletin officiel spécial nº 1 du 5 février 1987.

Ministère de l'Éducation nationale (2001). Encart Bulletin officiel $n^{\circ} 28$ du 12 juillet 2001. En ligne : https://www.education.gouv.fr/bo/2001/28/encart.htm.

Ministère de l'Éducation nationale (2002). Bulletin officiel $n^{\circ} 41$ du 7 novembre 2002. En ligne : https://www.education.gouv.fr/bo/2002/41/default.htm.

Ministère de l'Éducation nationale (2006). Bulletin officiel $n^{\circ} 40$ du 2 novembre 2006. En ligne : https://www.education.gouv.fr/bo/2006/40/default.htm.

Ministère de l'Éducation nationale (2015). « Programmes d'enseignement du cycle des apprentissages fondamentaux (cycle 2), du cycle de consolidation (cycle 3) et du cycle des approfondissements (cycle 4) ». Bulletin officiel spécial no 11 du 26 novembre 2015. En ligne : https:// www.education.gouv.fr/pid285/bulletin_officiel.html?pid_bo=33400.

Ministère de l'Éducation nationale (2018). « Projet d'ajustement et de clarification des programmes de français des cycles 2, 3 et 4 - Voté le 14 juin $2018 »$. Projets d'ajustement et de clarification des programmes de quatre enseignements pour la scolarité obligatoire (cycles 2, 3 et 4). En ligne : https://www.education.gouv.fr/projets-d-ajustement-et-de-clarification-desprogrammes-de-quatre-enseignements-pour-la-scolarite-8381.

Ministère de l'Éducation nationale (2019). Bulletin officiel spécial $n^{\circ} 1$ du 22 janvier 2019. En ligne : https://www.education.gouv.fr/pid285/bulletin_officiel.html?pid_bo=38502.

\section{NOTES}

1. Voir les références dans la section "Corpus de textes officiels » disponible en bibliographie. Il est à noter que certains textes officiels ne sont pas archivés en ligne. C'est notamment le cas des bulletins officiels antérieurs au 11 juin 1998, et c'est 
pourquoi certaines références ces textes n'ont pas d'URL en bibliographie. Plus de détail à ce sujet: https://eduscol.education.fr/numerique/dossier/archives/ ressources-en-ligne/lois-et-reglements/journaux-et-bulletins-officiels.

2. Sur la lecture analytique au collège voir par exemple Plissonneau, Bazile \& Boutevin (2017).

3. Ce terme peut aussi bien désigner l'explication de texte (Denizot, 2014), la lettre métatextuelle, (Daunay, 2019), la lecture littéraire (Dufays, 2016) ou l'extrait (Langlade, 2014 ; Brunel et al., 2019),

4. M. Charles (1985, p. 312) parle de " culture du commentaire».

5. S'y ajoute le commentaire, au baccalauréat à partir de 1969 (Daunay 2005 ; Denizot, 2013a).

6. L'hypertextualité étant conçue comme «toute relation unissant un texte B ([...] hypertexte) à un texte antérieur $A$ ([...] hypotexte) sur lequel il se greffe d'une manière qui n'est pas celle du commentaire » (Genette, 1982, p. 11-12).

\section{RÉSUMÉS}

Cet article interroge l'origine des tensions à l'œuvre dans la lecture analytique. Il adopte une perspective historico-didactique et se limite aux textes officiels français parus depuis le milieu du $\mathrm{XIX}^{\mathrm{e}}$ siècle. Il montre que ces tensions s'expliquent tout d'abord par l'approche méthodique des textes qui fonde l'exercice en 1880 , ce qui a pour conséquences une lecture métatextuelle, une place importante accordée à la méthodologie et la méfiance à l'égard des impressions personnelles. Ensuite, il montre que ces tensions existent dans les programmes eux-mêmes qui déplorent une lecture standardisée, sans pour autant remettre en question l'approche métatextuelle dans un exercice qui s'est réifié. Enfin, il explique que tensions sont aussi liées, à partir des années 1980, aux reconfigurations des théories de référence qui sous-tendent l'exercice, et à la place qu'occupent alors les théories du sujet lecteur dans un exercice qui continue de se réclamer d'une approche méthodique.

This article questions the origin of the tensions at work in analytical reading. It does so from a double perspective, historical and didactic and it is based on the French official bulletins that have been published since the middle of the $19^{\text {th }}$ century. It shows these tensions can be explained by the methodic approach of texts that dates back to 1880 when the exercise was created. It led to metatextual reading, gave a prominent place to methodology and led to mistrust with regard to personal impressions. Then, it shows these tensions are present in the curriculums themselves: standardized reading is disapproved of, but the metatextual approach has not actually been brought into question for this exercise that has fossilized. Finally, it shows that from the 1980s onwards, these tensions have also been connected to the reconfigurations of theoretical frameworks for the exercise and to the reader response theories for an exercise that is supposedly based on a methodic approach. (Traduction du résumé par Carole Couegnas, IUT Angoulême). 
INDEX

Mots-clés : Explication de texte, Lecture analytique, Lecture méthodique, Exercice, Avatar, Historico-didactique, Métatexte, Histoire littéraire, Sujet lecteur

Keywords : Textual analysis, analytical reading, methodical reading, Exercise, Avatar, historical an didactic research, Literary history, reader response theory

\section{AUTEUR}

\section{LAETITIA PERRET}

Université de Poitiers, Laboratoire FoReLLIS, F-86073 Poitiers, France 